\title{
Theory for an electrostatic imaging mechanism allowing atomic resolution of ionic crystals by atomic force microscopy
}

\author{
F. J. Giessibl \\ IBM Research Division, Physics Group Munich, Schellingstrasse 4, D-W8000 München 40, Germany
}

(Received 9 March 1992)

\begin{abstract}
An electrostatic imaging mechanism is presented which allows atomic resolution of the surface of ionic crystals by atomic force microscopy (AFM). In the $x-y$ plane the electrostatic field due to the ion charges reflects the periodicity of the surface lattice. If the tip of the AFM stylus is polarizable, an attractive force between tip and sample will exist and allow imaging of the surface in a noncontact mode. It is shown that the decay length of the electrostatic interaction in the $z$ direction is sufficiently short for atomic resolution to be achieved not only with a hypothetical tip consisting of only one atom but also by a more realistic tip of parabolic shape with a radius of $30 \mathrm{~nm}$. The theory is applied to the (001) surface of $\mathrm{KBr}$.
\end{abstract}

The atomic force microscope (AFM) is a tool which allows the study of nonconducting surfaces on the atomic scale. ${ }^{1}$ In order to obtain atomic resolution, the interaction between the AFM tip and the sample needs to be very strongly distance dependent, so that the largest contribution to the lateral-dependent part of the force between tip and sample is that between the topmost tip atom and the surface (see Fig. 1). Atomic resolution by AFM has so far only been reported in the repulsive mode, i.e., the force between the topmost atom of the AFM tip and the sample is repulsive. Albrecht and Quate ${ }^{2}$ first succeeded in imaging a nonconductor (boron nitride) and calculated the contrast by using the Gordon-Kim potential ${ }^{3}$ which describes the repulsive interaction of two closed-shell atoms when brought very close together. At the equilibrium distance of two ions with closed shells, the repulsive force according to Gordon and Kim increases by a factor of 100 when the distance is decreased by $0.1 \mathrm{~nm}$. Several groups have succeeded in atomic resolution of ionic crystals. Meyer et al. ${ }^{4}$ have achieved atomic resolution of the $\mathrm{F}^{-}$ sublattice on $\mathrm{LiF}(001)$, while Meyer and $\mathrm{Amer}^{5}$ have imaged the $\mathrm{Cl}^{-}$sublattice of $\mathrm{NaCl}(001)$ on the atomic scale. Meyer et al. ${ }^{6}$ have achieved atomic resolution on

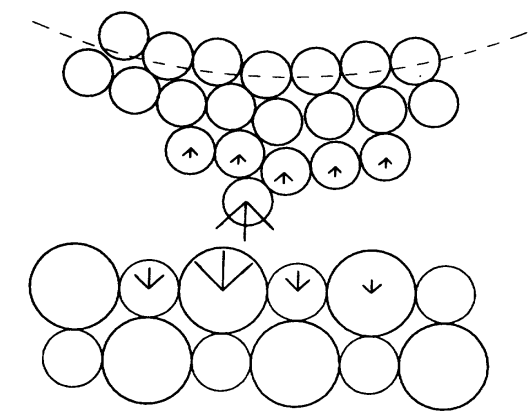

FIG. 1. Microscopic view of AFM tip and sample. The length of the arrows indicates the strength of the interaction between the individual atoms. From this figure it is clear that in order to get atomic resolution the interaction must be very strongly distance dependent. epitaxially grown films of $\mathrm{AgBr}(001)$ in air. Our group has been able to image the $\mathrm{K}^{+}$and the $\mathrm{Br}^{-}$ions of a (001) KBr surface. ${ }^{7}$

In vacuum, the attractive force between an AFM tip and a sample is typically dominated by a large van der Waals force. The magnitude of this force can be calculated by summing the interactions of each individual tip atom with each atom of the sample. However, the shape of the tip is generally unknown and even if it were, the calculation would be hard to do and the conclusion would only be valid for a certain tip geometry. One can split the van der Waals force into a part that does not depend on the lateral position of the tip and another part that does depend on the lateral position.

Girard, Van Labeke, and Vigoureux ${ }^{8}$ have calculated that the van der Waals force between a tungsten probe of a radius $r=0.2 \mathrm{~nm}$ and a $(001) \mathrm{NaCl}$ surface is larger by $0.257 \mathrm{nN}$ when the probe is on top of $\mathrm{Cl}^{-}$ion than when on top of a $\mathrm{Na}^{+}$ion. Table VII in Ref. 8 shows that the laterally dependent part of the van der Waals force decays exponentially at a rate of $10^{-z / 0.1 \mathrm{~nm}}$.

In this paper it is shown that the electrostatic contribution to the force between a polarizable tip and the surface of an alkali halide also decays at a rate of approximately

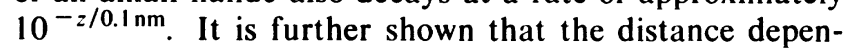
dence of the laterally dependent van der Waals force and the electrostatic force is sufficiently strong for the contribution of the topmost tip atom to be larger than the contribution of the rest of the tip. This important issue will be proven later.

The part of the van der Waals force that does not depend on the lateral position of the tip ("background force") is in general 2 to 4 orders of magnitude larger than the part that does depend on lateral position. In general, in all the atomically resolved AFM pictures, the total force on the AFM tip was attractive, but the force on the topmost tip atom was repulsive. The tip atom can only transmit a force up to a certain limit, depending on tip material and rigidity of the sample. Therefore, the largest part of the background force has to be counteracted by the spring that holds the AFM tip. The sample would be disturbed to a significantly lesser degree if one could im- 


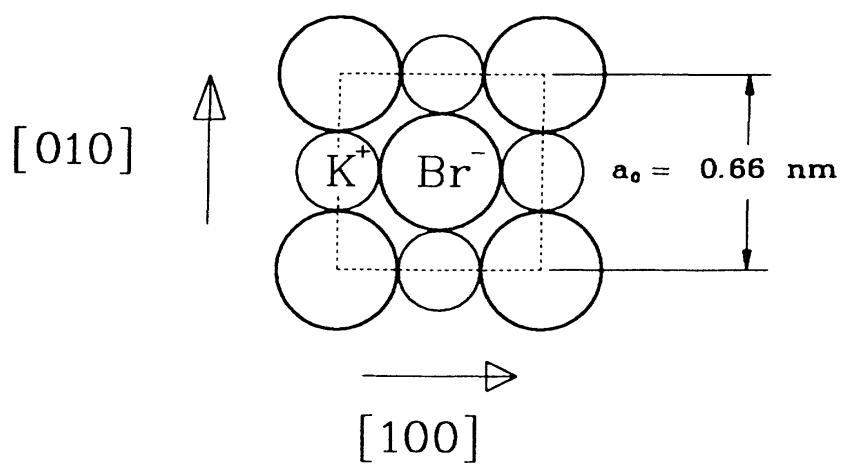

FIG. 2. Surface structure of $\mathrm{KBr}(001)$. $\mathrm{KBr}$ crystallizes into an fcc lattice with a lattice constant of $0.66 \mathrm{~nm}$. The large circles represent the $\mathrm{Br}^{-}$ions (bare ion radius $0.195 \mathrm{~nm}$ ), and the small circles represent the $\mathrm{K}^{+}$ions (bare ion radius $0.133 \mathrm{~nm}$ ).

age in the attractive mode; i.e., if the net force between tip atom and surface were attractive.

In this paper it is shown that the electrostatic force which arises between the surface of an alkali halide and a polarizable tip should allow atomic resolution. To an excellent approximation alkali halides can be seen as consisting of hard spheres which are charged by plus or minus one unit charge. ${ }^{9}$ Many of them crystallize into an fcc lattice with a two-atom basis (the anion at zero and the cation displaced half a lattice constant in a $\langle 100\rangle$ direction). The calculation presented here is based on the $\mathrm{KBr}$ lattice parameters, but it could be adapted to other crystal lattices as well. Figure 2 shows the structure of the $\mathrm{KBr}(001)$ surface.

The electrostatic field at the $(001)$ surface of $\mathrm{KBr}$ was calculated numerically by using the superposition principle. A crystallite composed of $81 \times 81 \times 81$ nonprimitive cubic unit cells was used as a model. The individual contribution of each ion to the electric field was then summed

$E(r)=\frac{e}{4 \pi \epsilon_{0}}\left[\sum_{\substack{\text { anion }- \\ \text { sites } \alpha}} \frac{r}{\left|r-r_{\alpha}\right|^{3}}-\sum_{\begin{array}{c}\text { cation }- \\ \text { sites } \kappa\end{array}} \frac{r}{\left|r-r_{\kappa}\right|^{3}}\right]$.

Figure 3 shows the $z$ dependence of the $z$ component of

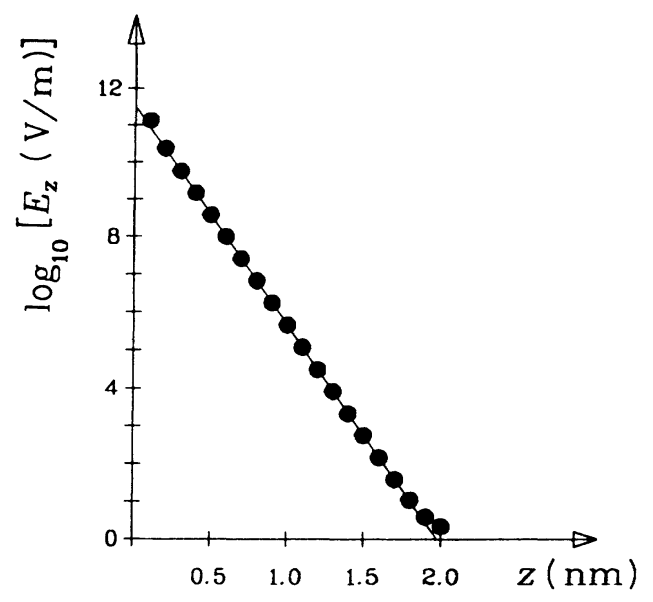

FIG. 3. Magnitude of the electrostatic field on top of a $\mathrm{K}^{+}$ ion. The field was calculated by summing the contributions of all the individual ions of a crystallite composed of $81 \times 81 \times 81$ cubic unit cells. Due to the finite size of the crystallite, the values at $z=1.9$ and $2 \mathrm{~nm}$ deviate slightly from being exponential.

the field. In the case of $\mathrm{KBr}$ the magnitude of the field increases by a factor of 10 when the distance is decreased by $0.18 \mathrm{~nm}$. To an excellent approximation, the magnitude of the field decays at a rate proportional to $\exp -k z$, where $k=2 \pi / a_{0}{ }^{10}$

The tip of the cantilever is polarized by this electrostatic field, as shown in Fig. 4. The magnitude of the induced dipole moment is given by

$$
p_{\mu}=\alpha \epsilon_{0} E_{\mu}
$$

where $\alpha$ is the electrostatic polarizability and $\epsilon_{0}$ is the electrostatic field constant. The interaction of this dipole with the electrostatic field causes a force in the $z$ direction given by

$$
F_{z}=p_{x} d E_{z} / d x+p_{y} d E_{z} / d y+p_{z} d E_{z} / d z .
$$

The magnitude of the dipole moment is proportional to the strength of the electrical field. The $z$ component of the force acting on the tip is given by the scalar product of the

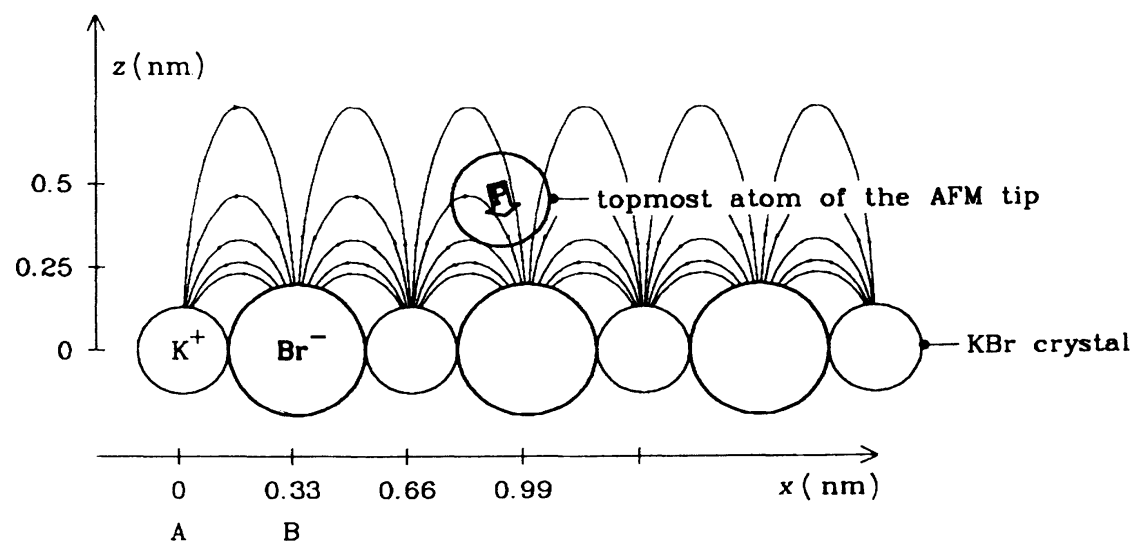

FIG. 4. Topmost atom of the AFM tip in the electrostatic field of the crystal. 
dipole moment and the gradient of the $z$ component of the electrostatic field. Thus the force is proportional to the derivative of the square of the electrostatic field strength. The force between tip and sample decreases to one tenth upon an increase in distance of $0.09 \mathrm{~nm}$ (for $\mathrm{KBr}$ ). Thus the distance dependence of the electrostatic force is about the same as that of the tunneling current for metallic electrodes.

If one tries to verify imaging in the attractive mode, it is helpful to use an AFM tip with a very small tip radius in order to keep the background force as small as possible. Two groups make cantilevers with very sharp tips, both made of silicon. ${ }^{11,12}$ Since the surface of silicon oxidizes in air, it seems reasonable to assume that the topmost tip atom is oxygen. Thus the maximum attractive force between the tip and sample at a minimal distance of $0.34 \mathrm{~nm}$ is $F_{\text {dip }}=5.5 \times 10^{-12} \mathrm{~N}$ if the tip is on top of either a $\mathrm{K}^{+}$or a $\mathrm{Br}^{-}$ion (assuming polarizability of $\mathrm{O}^{2-}=3.88 \times 10^{-30}$ $\mathrm{m}^{3},{ }^{13}$ electrical field strength $E_{z}=E_{0} \exp [-z / 0.0738$ $\mathrm{nm}$, and $\left.E_{0}=5 \times 10^{11} \mathrm{~V} / \mathrm{m}\right)$. The electrostatic field for constant height $z$ is approximately given by $E_{z}$ $=E_{0} \cos \left(2 \pi x / a_{0}\right) \cos \left(2 \pi y / a_{0}\right)$, with a cubic lattice constant $a_{0}=0.66 \mathrm{~nm}$. Figure 5 shows the force between the oxygen atom and the $\mathrm{KBr}$ surface for three different heights along the path $A-B$.

This model includes three major simplifications: (a) the nonzero size of the tip atom is neglected; (b) the influence of the tip atom on the electrostatic field of the surface is neglected; and (c) the polarizability of the oxygen ion is assumed to be constant despite the large magnitude of the field and the field gradient.

The experimental verification of imaging ionic crystals in the attractive mode is to our knowledge still lacking. A striking effect should occur when switching from repulsive

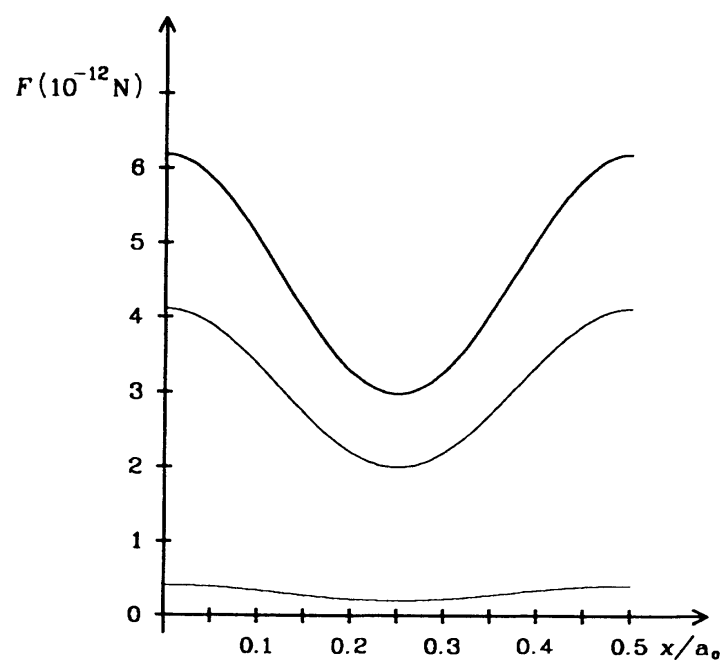

FIG. 5. Attractive electrostatic force between an oxygen atom and a $\mathrm{KBr}$ surface plotted along the path $A-B$ (see Fig. 4). The magnitude of the force is the same over a $\mathrm{K}$ site as over a $\mathrm{Br}$ site. The lower curve corresponds to a distance (between the center of the oxygen atom and the center of the surface ions) of $0.435 \mathrm{~nm}$, the distance in the middle curve is $0.35 \mathrm{~nm}$, and for the curve at the top the distance is $0.335 \mathrm{~nm}$. imaging to attractive imaging. When imaging the van der Waals forces, the halide ions will appear as depressions, i.e., the strongest attraction of the AFM tip will occur at the halide sites. When imaging the electrostatic forces, both ionic species will appear as depressions, because the attractive force is maximal on top of either ion site.

In repulsive imaging, the AFM tip will move from the sample at the sites of ions. Thus the ions will appear as bright spots in the repulsive mode and dark spots in the attractive mode. The laterally dependent part of the van der Waals force between a tungsten tip (diameter $0.2 \mathrm{~nm}$ ) and a (001) $\mathrm{NaCl}$ surface calculated by Girard, Van Labeke, and Vigoureux ${ }^{8}$ is 2 orders of magnitude larger than the electrostatic force for an oxygen atom as an AFM tip and the (001) surface of $\mathrm{KBr}$. However, the periodicity of the van der Waals force is $1 / a_{0}$, while that of the electrostatic interaction is $2 / a_{0}$. It should therefore be possible to distinguish between the contributions of the van der Waals and the electrostatic force.

It remains to be shown that not only a tip consisting of one atom, but also a macroscopic tip will give atomic resolution in the attractive mode. Figure 6 shows a model of a macroscopic tip. The cross section of the tip is assumed to be a parabola with a radius of curvature $\rho$. For simplicity we assume a simple-cubic crystal structure with lattice constant $a$ for the tip material and the [001] direction aligned with the symmetry axis. The number of atoms $N$ in plane $n$ at height $z=a n$ is thus given by the area of the plane divided by the cross section of a unit cell,

$$
N(z)=2 \pi \rho z / a^{2} .
$$

Substituting $z=$ an yields

$$
N(n)=2 \pi \rho n / a \text { for } n>0 \text { and } N(0)=1 .
$$

The distance dependence of the laterally dependent part of the force between an atom and the surface is given by

$$
F(z)=F_{0} e^{-z / \zeta}
$$

with $\zeta=0.035 \mathrm{~nm}$. The total force between tip and sample for a distance $z$ between the apex of the tip and the surface is thus given by

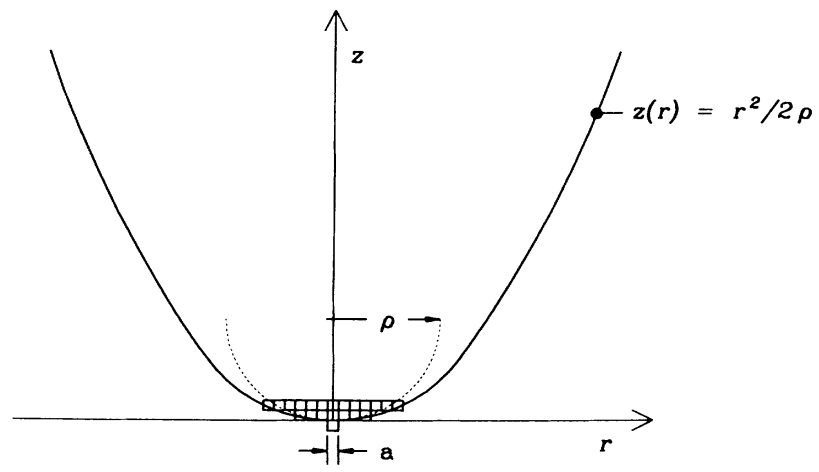

FIG. 6. Model for the AFM tip with a parabolic cross section. The crystal structure of the tip is assumed to be simple cubic. The number of atoms per plane can thus be calculated by dividing the area of each plane by the size of the unit cell. 


$$
F(z)=F_{0} e^{-z / \zeta}\left(1+2 \pi \rho / a \sum_{n} n e^{-z n / \alpha}\right),
$$

where $\sum_{n}$ means the sum for $n$ from zero to infinity. Using the identity

$$
\begin{aligned}
\sum_{n} n e^{-\lambda n} & =-d / d \lambda\left(\sum_{n} e^{-\lambda n}\right)=-d / d \lambda\left(1-e^{-\lambda}\right)^{-1} \\
& =e^{-\lambda /\left(1-e^{-\lambda}\right)^{2}}
\end{aligned}
$$

and assuming $a=0.3 \mathrm{~nm}(\lambda=0.3 / 0.037=8.1)$ yields

$$
F(z)=F_{0} e^{-z / \zeta}\left(1+2 \pi \rho / a e^{-8.1}\right) .
$$

The ratio of the force between the topmost tip atom and the whole tip is thus given by

$$
F_{\text {tip atom }} / F_{\text {total }}=1 /(1+0.0019 \rho / a) .
$$

For a tip of radius $\rho=30 \mathrm{~nm}$, Eq. (9) implies that $84 \%$ of the force between tip and sample is transmitted by the topmost tip atom. Therefore it is proven that even a realistically shaped tip can produce atomic resolution by electrostatic interaction.

I would like to thank Douglas Smith for encouraging me to publish this work and Gerd Binnig for useful discussions.
'G. Binnig, C. F. Quate, and Ch. Gerber, Phys. Rev. Lett. 56, 930 (1986).

${ }^{2}$ T. R. Albrecht and C. F. Quate, J. Appl. Phys. 62, 2599 (1987).

${ }^{3}$ R. G. Gordon and Y. S. Kim, J. Chem. Phys. 56, 3122 (1972).

${ }^{4}$ E. Meyer, H. Heinzelmann, H. Rudin, and H.-J. Güntherodt, Z. Phys. B 79, 3 (1990).

${ }^{5}$ G. Meyer and N. M. Amer, Appl. Phys. Lett. 56, 2100 (1990).

${ }^{6}$ E. Meyer, H.-J. Güntherodt, H. Haefke, G. Gerth, and M. Krohn, Europhys. Lett. 15, 319 (1991).

${ }^{7}$ F. J. Giessibl and G. Binnig, Ultramicroscopy (to be published).
${ }^{8}$ C. Girard, D. Van Labeke, and J. M. Vigoureux, Phys. Rev. B 40, 12133 (1989).

${ }^{9}$ N. W. Ashcroft and N. D. Mermin, Solid State Physics (Saunders College, Philadelphia, 1976), p. 402.

10 J. E. Lennard-Jones and B. M. Dent, Trans. Faraday Soc. 24, 92 (1928).

I'S. Akamine, R. C. Barrett, and C. F. Quate, Appl. Phys. Lett. 57, 316 (1990).

${ }^{12} \mathrm{O}$. Wolter, Th. Bayer, and J. Greschner, J. Vac. Sci. Technol. B 9, 1353 (1991).

${ }^{13}$ C. Kittel, Introduction to Solid State Physics, 6th ed. (Wiley, New York, 1986), p. 371. 